\title{
STEADY STATE RESPONSE AND STABILITY OF AN ELASTICALLY RESTRAINED TAPERED BEAM
}

\author{
M.S. Abdel-Jaber ${ }^{1}$, A.A. Al-Qaisia ${ }^{2, *}$, M. Abdel-Jaber ${ }^{3}$ and R.G. Beale ${ }^{4}$ \\ ${ }^{1}$ Associate Professor, Department of Civil Engineering, The University of Jordan, Amman, Jordan \\ ${ }^{2}$ Professor, Department of Mechanical Engineering, The University of Jordan, Amman, Jordan \\ ${ }^{3}$ Associate Professor, Department of Civil Engineering, Faculty of Engineering and Technology, \\ Applied Science University, Amman, Jordan \\ ${ }^{4}$ Reader, Department of Mechanical Engineering and Mathematical Sciences, \\ Oxford Brookes University, Oxford, United Kingdom \\ *(Corresponding author: E-mail: alqaisia@ju.edu.jo)
}

Received: 4 June 2012; Revised: 2 July 2012; Accepted: 8 October 2012

\begin{abstract}
An analytical method for the study of the nonlinear forced vibrations and their stabilities of an elastically restrained tapered cantilever beam due to a direct periodic excitation is developed. The method of harmonic balance is used to study the steady state frequency response of the beam system for different values of physical parameters such as the root translational and rotational stiffness and the beam taper ratio. Results are presented for the first three modes of vibration. The stability of the frequency response for some selected values of the physical parameters is investigated, i.e. the regions on the frequency response curves at which the solution may bifurcate and then culminate into chaos. The qualitative features of the solutions are studied and identified using phase plane, Poincare maps and Fast Fourier Transform. The results are presented, discussed and conclusions on the elastically restrained tapered beam nonlinear dynamics are drawn.
\end{abstract}

Keywords: Forced vibration, tapered beam, elastically restrained, stability, period doubling, chaos

\section{INTRODUCTION}

Beams with continuous changes in cross sectional area along with the beam axis, i.e. tapered beams, have been extensively used in many civil and mechanical engineering structures such as; such as offshore structure piles, oil platform supports, oil-loading terminals, tower structures, bridges, lighting standards, rotating tapered blades and moving/rotating arms. Tapered beams are also increasingly being used in the construction industry, due to their particular specifications and unique ability to combine efficiency, economy and aesthetic architectural needs. They have the capability to optimise weight and strength despite the cost of fabrication of tapered members.

Since tapered beams can model many engineering structures that require variable stiffness along the beam axis "length", designs and analyses of such structures have attracted considerable interest and many researchers have directed their efforts and much research have been devoted towards the mathematical modelling and dynamic behaviour.

Studying the dynamic analysis and vibrational behaviour of tapered beams enables the predetermination of undesirable behaviour such as resonance, large vibration levels and unstable vibration behaviour. The results of which will help designers and engineers predict the dynamic behaviour and enable them to impose suitable vibration control strategies to achieve optimum and safe behaviour.

Having realized the vital role of the accurate mathematical modelling of tapered beams and having recognized their importance in studying dynamic behaviour researchers published many studies and reported analysis to deal with calculating natural frequencies and modes shapes either for small or large amplitude vibrations. Broad surveys and literature on these subjects can be found in [1-14]. 
In fact, most of the previous research in this direction has been oriented towards the calculation of linear natural frequencies and mode shapes, with different end conditions and with attached inertia elements at the free end of the beam.

In general, tapered beams may have relatively high flexibility due to their high aspect ratio and as they are usually subjected to various excitation loads such as wind loads, wave loads, etc., the prediction of their steady state response, nonlinear forced vibrations and stability of these large amplitude vibrations is extremely important for design, control and analysis.

The objective of the present work is to extend the analysis and the results obtained in $[13,14]$ by studying the non-linear, planar, large amplitude forced vibrations of an elastically restrained tapered beam for the cases of a double taper beam and a single taper "wedge shaped beam". The mathematical model is derived using the Lagrange method and the resulting continuous equation is discretised using the assumed mode method. The inextensibility condition [14-17] is used to determine the axial shortening due to transverse deflection in the formulation of the kinetic energy of the beam and the nonlinear curvature is used in the potential energy expression.

Abdel-Jaber et al. $[13,14]$ undertook a complete study into the non-linear natural frequencies of an elastically restrained tapered cantilever. Their results documented a parametric study into the frequency-amplitude nonlinear relation under different physical parameters and combinations. The tapered beam system results have shown that, for the first and second modes the behaviour is changed from a hardening type to a softening type when the taper ratio is increased, whilst the third mode is always of a softening type regardless the value of the taper ratio (a hardening behaviour leads to an increase in the natural frequency of the beam when the vibration amplitude increases and a softening behaviour to a decrease in natural frequency). For a given value of taper ratio the authors showed that the nonlinear natural frequency of a double tapered beam is higher than that of a single tapered beam. The authors also produced results for the effect of the stiffness of the connection between the cantilever and the ground on the nonlinear natural frequency; it was shown that the nonlinear natural frequency changes from a softening to a hardening behaviour depending upon combinations of the physical parameters of the connection stiffness and the vibration amplitude.

As the physical properties of cantilever beam systems such as connection stiffness may change during operation studying the steady state response under periodic excitation is extremely important.

In the light of the above review, the objective of this work is to extend the analyses in $[13,14]$ and to analyze the periodic steady state response of the nonlinear oscillator which describes large amplitude planar vibrations of an elastically restrained tapered beam, under different values of root translational and rotational flexibilities. Furthermore, the stability of the obtained solutions is investigated and discussed to determine for a selected range of system parameters the regions of period doubling bifurcations and chaos on frequency response curves.

To the authors' knowledge, despite its physical importance, studies dealing with forced vibrations and their stability of tapered beams are not commonly available. 


\section{DERIVATION OF THE EQUATION OF MOTION}

For the system shown in Figure 1 a complete derivation of the equation of motion is presented in [14] and for the sake of brevity it will not be presented here.

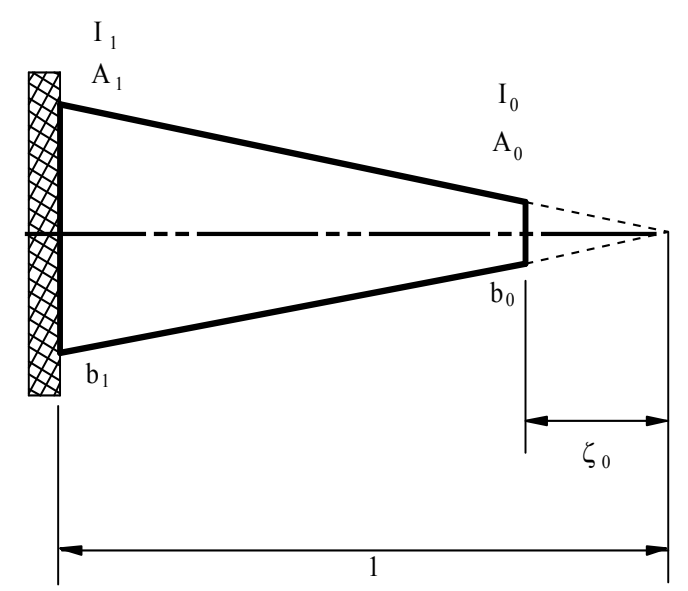

(a) Schematic for the tapered beam

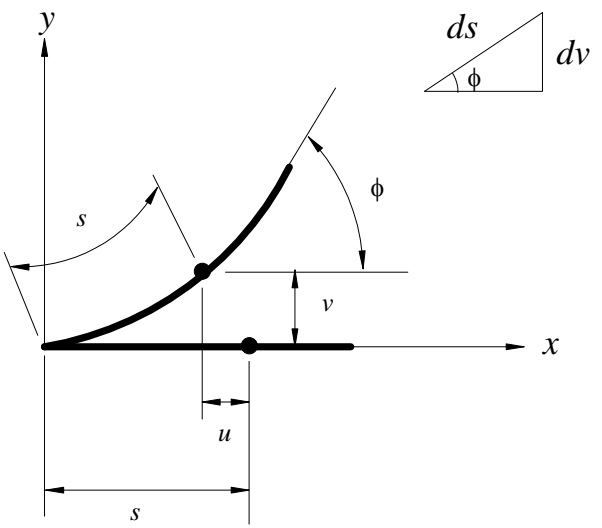

(b) The deformed inextensible beam

Figure 1. Beam Schematics

The rotational and translational stiffnesses of the elastic restraint at the cantilever root are denoted by $K_{r}$ and $K_{t}$ respectively. The Lagrangian of the system under consideration is given by:

$$
L=\rho l^{3}\left(\beta_{1} \dot{q}^{2}+\beta_{2} q^{2} \dot{q}^{2}-\beta^{2} \beta_{3} q^{2}-\beta^{2} \beta_{4} q^{4}\right)
$$

where

$$
\beta_{1}=\int_{0}^{1} A_{1}^{*} \phi^{2} \mathrm{~d} \zeta
$$

$\beta_{2}=\int_{0}^{1} A_{1}^{*}\left\{\int_{0}^{\zeta} \phi^{\prime 2} \mathrm{~d} \chi\right\}^{2} \mathrm{~d} \zeta$

$\beta_{3}=\int_{0}^{1} I_{1}^{*} \phi^{\prime \prime 2} \mathrm{~d} \zeta+\frac{K_{t} l^{3}}{E I_{1}} \phi(1)^{2}+\frac{K_{r} l}{E I_{1}} \phi^{\prime}(1)^{2}$

$\beta_{4}=\int_{0}^{1} I_{1}^{*} \phi^{\prime 2} \phi^{\prime 2} \mathrm{~d} \zeta$

$\phi(\zeta)$ is the normalized, self-similar (i.e. independent of the motion amplitude) assumed mode shape of the beam and $q(\hat{t})$ is an unknown time modulation of the assumed deflection mode $\phi(\zeta)$. A dot is used to denote a derivative with respect to the non-dimensional time.

For a double tapered beam $A_{1}^{*}=A_{1} \zeta^{2}$ and $I_{1}^{*}=I_{1} \zeta^{4}$ and for a single tapered wedge beam $A_{1}^{*}=A_{1} \zeta$ and $I_{1}^{*}=I_{1} \zeta^{3}$. As can seen $\beta_{i}, i=1, . ., 4$ are functions of the system physical parameters, so that any variation in any physical quantity such as the root flexibility will be reflected in the calculated values of $\beta_{i}$. 
To study the forced planar response of the beam system, a periodic excitation $Q(\hat{t})$ is assumed to act only in the $y$ direction, the beam transverse direction. Upon the application of the Euler-Lagrange equation

$Q=\frac{\partial}{\partial \hat{t}}\left(\frac{\partial L}{\partial \dot{q}}\right)-\frac{\partial L}{\partial q}$

where $Q$ is the generalized force, which can be determined from the principle of virtual work, $(\delta W=Q \cdot \delta y)$. Assuming $Q(?)=\bar{F}_{0} \cos (\Omega t)$, the discrete beam nonlinear equation becomes

$\beta_{1} \ddot{q}+\beta^{2}\left(\beta_{3} q+2 \beta_{4} q^{3}\right)+\beta_{2}\left(q^{2} \ddot{q}+q \dot{q}^{2}\right)=\beta_{5} \bar{F}_{0} \cos (\Omega \hat{t})$

where $\beta_{5}=\phi\left(\zeta_{c}\right)$ for a concentrated load which is assumed to act at an arbitrary point $\zeta_{c}$ along the beam span and $\beta_{5}=\int_{\chi}^{1} \phi(\zeta) \mathrm{d} \zeta$ for a distributed load acting from $\chi$ to the end of the beam. It is to be noted that some of the coefficients $\beta_{i}$ in Eqs. 2-5, increase sharply and attain relatively large values at higher modes of the beam. Therefore, for convenience, Eq. 7 is scaled and converted to the dimensionless form

$\ddot{q}+q+\varepsilon_{1}\left(q^{2} \ddot{q}+q \dot{q}^{2}\right)+\varepsilon_{2} q^{3}=\varepsilon_{3} \bar{F}_{0} \cos (\Omega t)$

where $t=\left(\beta^{2} \beta_{3} / \beta_{1}\right)^{1 / 2} \hat{t}$ and $\varepsilon_{1}=\frac{\beta_{2}}{\beta_{1}}, \varepsilon_{2}=\frac{2 \beta_{4}}{\beta_{3}}$ and $\varepsilon_{3}=\frac{\beta_{5}}{\beta_{3}}$ are dimensionless coefficients.

For the stability analysis the damping of the beam system is assumed to be viscous, with damping coefficient $\delta$, which can be added to the system, also the excitation level for convenience is rescaled such that $\varepsilon_{3} \bar{F}_{0}=F_{0}$. The equation of motion takes the form:

$\ddot{q}+q+\delta \dot{q}+\varepsilon_{1}\left(q^{2} \ddot{q}+q \dot{q}^{2}\right)+\varepsilon_{2} q^{3}=F_{0} \cos (\Omega t)$

Eq. 9 describes the non-linear, planar, flexural free vibration of the inextensible elastically restrained tapered beam. In this equation, the terms $\varepsilon_{1} \ddot{q} q^{2}$ and $\varepsilon_{1} q \dot{q}^{2}$ are inertial non-linearities due to the kinetic energy of the axial motion which arise as a result of using the inextensibility condition and they are of a softening type. The non-linear term $\varepsilon_{2} q^{3}$ is due to the potential energy stored in bending and arises as a result of using non-linear curvature and it is of a hardening static type.

\section{ANALYSIS}

Approximate analytical solutions for the periodic steady state response, having the same period as the excitation, of the nonlinear oscillator described by Eq. 9 are obtained using the harmonic balance method (HB). To simplify the analysis a new time $T=\Omega t$ is introduced so that Eq. 9 becomes 
$\Omega^{2} \ddot{q}+\Omega \delta \dot{q}+q+\varepsilon_{1} \Omega^{2} q^{2} \ddot{q}+\varepsilon_{1} \Omega^{2} q \dot{q}^{2}+\varepsilon_{2} q^{3}=F_{0} \cos (T)$

where dots are derivatives with respect to the new time $T$.

\subsection{Steady State Solution, Harmonic Balance Method (HB)}

According to the Harmonic Balance method, an approximate single term solution (SHB) of Eq. 10 should contain sine and cosine terms, but with the same harmonic. To avoid this, an unknown phase $\phi$ can be introduced to the excitation, and Eq. 10 takes the form

$\Omega^{2} \ddot{q}+\Omega \delta \dot{q}+q+\varepsilon_{1} \Omega^{2} q^{2} \ddot{q}+\varepsilon_{1} \Omega^{2} q \dot{q}^{2}+\varepsilon_{2} q^{3}=F_{0} \cos (T+\phi)$

In this way, one may introduce a fundamental harmonic response containing a single trigonometric term only, which takes the form

$$
q(T)=A \cos T
$$

where $A$ is the steady state response amplitude. Substituting Eq. 12 into Eq. 11, neglecting any third harmonics which arise, and equating coefficients of first harmonics the following equations are obtained:

$$
\begin{aligned}
& \left(\frac{3}{4} \varepsilon_{2}-\frac{\varepsilon_{1}}{2} \Omega^{2}\right) A^{3}+\left(1-\Omega^{2}\right) A=F_{0} \cos \varphi \\
& -\Omega \delta A=F_{0} \sin \phi
\end{aligned}
$$

To eliminate the unknown phase $\phi$, the steady state frequency response is obtained by squaring and adding Eq. 13 to Eq. 14 and solving for $\Omega^{2}$, for a given value $A$.

The accuracy of the solution obtained from the SHB approximation can be improved by adding higher harmonics in the assumed solution given in Eq. 12. In this paper one more term is added to this equation, whereby the two-term approximation (2THB) to the steady state solution of the Eq. 11 with odd nonlinearities in the form

$q(T)=A_{1} \cos T+A_{3} \cos 3 T+B_{3} \sin 3 T$

Substituting Eq. 15 into Eq. 12 and following the same procedure, i.e. neglecting higher-order harmonics, the following coupled nonlinear algebraic equations for $A_{1}, A_{3}, B_{3}$ and the phase $\phi$ are obtained:

$$
\begin{aligned}
& \frac{3}{4} \varepsilon_{2} A_{1}^{3}+\frac{3}{4} \varepsilon_{2} A_{1}^{2} A_{3}+\frac{3}{2} \varepsilon_{2} A_{1} A_{3}^{2}+\frac{3}{2} \varepsilon_{2} A_{1} B_{3}^{2}+A-A \Omega^{2}-\frac{\varepsilon_{1}}{2} \Omega^{2} A^{3} \\
& -\frac{3}{2} \varepsilon_{1} \Omega^{2} A_{1}^{2} A_{3}-5 \varepsilon_{1} \Omega^{2} A_{1}^{2} A_{3}-5 \varepsilon_{1} \Omega^{2} A_{1}^{2} B_{3}=F_{0} \cos \phi \\
& \frac{3}{4} \varepsilon_{2} A_{1}^{2} B_{3}-\Omega \delta A_{1}-\frac{3}{2} \varepsilon_{1} \Omega^{2} A_{1}^{2} B_{3}=F_{0} \sin \phi
\end{aligned}
$$




$$
\begin{aligned}
& \frac{3}{2} \varepsilon_{2} A_{1}^{2} B_{3}+\frac{3}{4} \varepsilon_{2} A_{3}^{2} B_{3}+\frac{3}{4} \varepsilon_{2} B_{3}^{3}+B_{3}-3 A_{3} \delta \Omega-9 \Omega^{2} B_{3}+ \\
& -5 \varepsilon_{1} \Omega^{2} A_{1}^{2} B_{3}-\frac{9}{2} \varepsilon_{1} \Omega^{2} A_{3}^{2} B_{3}-\frac{9}{2} \varepsilon_{1} \Omega^{2} B_{3}^{3}=0 \\
& \frac{\varepsilon_{2}}{4} A_{1}^{3}+\frac{3}{2} \varepsilon_{2} A_{1}^{3} A_{3}+\frac{3}{4} \varepsilon_{2} A_{3}^{2}+\frac{3}{4} \varepsilon_{2} A_{3} B_{3}^{2}+A_{3}+3 B_{3} \delta \Omega-9 A_{3} \Omega^{2}+ \\
& -\frac{\varepsilon_{1}}{2} \Omega^{2} A_{1}^{3}-5 \varepsilon_{1} \Omega^{2} A_{1}^{2} A_{3}-\frac{9}{2} \varepsilon_{1} \Omega^{2} A_{3}^{2}-\frac{9}{2} \varepsilon_{1} \Omega^{2} A_{3} B_{3}^{2}=0
\end{aligned}
$$

A steady state solution of the system can be obtained by solving the nonlinear equations given in Eq. 12 and Eq. 13 for the SHB and by the nonlinear equations given in Eqs. 16-19 for the 2THB using a numerical procedure. For the sake of brevity, a detailed procedure for solving such nonlinear systems is not given in this paper but is presented in [15-17].

\section{STABILITY OF STATE SOLUTIONS}

The stability analysis of the approximate harmonic balance solutions given in Eq. 11 and Eq. 14 may be carried out by introducing a small perturbation $v(T)$ to the assumed solution in Eq. 12 by substituting

$$
u(T)=A \cos T+v(T)
$$

into Eq. 10. This leads to the following non-linear variational equation

$$
\begin{aligned}
& \ddot{v} \Omega^{2}\left(1+\varepsilon_{1} \frac{A^{2}}{2}+\varepsilon_{1} v^{2}+2 \varepsilon_{1} v A \cos T+\varepsilon_{1} \frac{A^{2}}{2} \cos 2 T\right)+ \\
& \dot{v}\left(\delta \Omega-2 \varepsilon_{1} \Omega^{2} A v \sin T-\varepsilon_{1} \Omega^{2} A^{2} \sin 2 T\right)+ \\
& v\left(\frac{3}{2} \varepsilon_{2} A^{2}+1-\varepsilon_{1} \Omega^{2} \frac{A^{2}}{2}+\varepsilon_{1} \Omega^{2} \dot{v}^{2}+\frac{3}{2} \varepsilon_{2} A^{2} \cos 2 T-\frac{3}{2} \varepsilon_{1} \Omega^{2} A^{2} \cos 2 T\right)+ \\
& \varepsilon_{1} \Omega^{2} A^{2} \dot{v}^{2} \cos T+v^{2} A \cos T\left(3 \varepsilon_{2}-\varepsilon_{1} \Omega^{2}\right)+\varepsilon_{2} v^{3}=\frac{A^{2}}{4}\left(2 \varepsilon_{1} \Omega^{2}-\varepsilon_{2}\right) \cos 3 T
\end{aligned}
$$

The stability is governed by the linearised version of Eq. 21 . In addition, the excitation term on the right-side is deleted, because it has no influence on stability, this leads to the following Hill's type equation:

$$
\begin{aligned}
& \ddot{v} \Omega^{2}\left(1+\varepsilon_{1} \frac{A^{2}}{2}(1+\cos 2 T)\right)+\dot{v}\left(\delta \Omega-\varepsilon_{1} \Omega^{2} A^{2} \sin 2 T\right)+ \\
& v\left(1+\frac{A^{2}}{2}\left(3 \varepsilon_{2}-\varepsilon_{1} \Omega^{2}\right)+\frac{3}{2} A^{2} \cos 2 T\left(\varepsilon_{2}-\varepsilon_{1} \Omega^{2}\right)\right)=0
\end{aligned}
$$

By virtue of Floquet theory, a particular solution of the linearised variational equation, is sought in the form [17]: 
$v(T)=e^{\beta T} \eta(T)$

where $\beta$ is defined as the characteristic exponent and $\eta(T)$ is a periodic function with periods $T$ and $T / 2$ since the dimensionless frequencies of the sine and cosine terms in Eq. 22 are 2, this introduce the periods $\mathrm{T}$ and $\mathrm{T} / 2$. The solution of $v(T)$ is stable (respectively, unstable) if the real part of $\beta$ is negative (positive); and the real part of $\beta$ is zero on the boundary between stable and stable regions. In addition, some results are presented for the case of asymmetric solution and their stability by substituting

$u(T)=A_{0}+A_{1} \cos T+v(T)$

where $A_{0}$ is a constant bias and $A_{1}$ is the amplitude. The results are of importance because they give good indication about the region at which the steady state solution bifurcates and causes period doubling and then chaotic behaviour.

For a full detailed procedure regarding the stability analysis either using single term as the one assumed in Eq. 20, two terms or biased solution as in Eq. 24, the readers are invited to consult reference [17].

\section{RESULTS AND DISCUSSION}

The steady state frequency response of the non-linear, single mode, temporal equation of motion Eq. 9 of the elastically restrained tapered beam as shown in Figure 1, was calculated analytically using the harmonic balance method and with an excitation level $F_{0}$, for different values of the physical system parameters $K_{r}, K_{t}, \alpha, \delta$ where $K_{r}$ is the rotational stiffness and $K_{t}$ is the translational stiffness of the elastic restraint at the cantilever root; $\alpha$ is the taper ratio defined by $\alpha=\frac{b_{1}}{b_{2}}$ and $\delta$ is the damping coefficient. Here and for the sake of convenience the beam root stiffnesses are introduced in terms of their flexibilities, $C_{r}=E I_{1} / K_{r} l$ and $C_{t}=E I_{1} / K_{t} l^{3}$. For example, for a clamped end $C_{r}=C_{t}=0$.

To calculate the parameters $\varepsilon_{1}$ and $\varepsilon_{2}$ given in the nonlinear Eq. 9, for selected values of the physical parameters, the integrals in Eqs. 2-5 defining the coefficients $\beta_{i}$ were evaluated numerically.

The results show that for a given value of $\alpha$ the nonlinear natural frequency of a double tapered beam is higher than that of a single tapered beam. In addition, the effect of the beam's root flexibility, was shown in [14] to have a significant role in the behaviour and dynamics of the beam, i.e. the behaviour was changed form a softening to a hardening behaviour depending on combinations of the physical parameters of the beam's root flexibilities $C_{r}$ and $C_{t}$.

The nonlinear frequency response of the beam due to excitation are dominated by the two competing non-linearities - softening " $\varepsilon_{1}\left(q^{2} \ddot{q}+q \dot{q}^{2}\right)$ " and hardening " $\varepsilon_{2} q^{3}$ " nonlinearities, and the behaviour of the elastically restrained tapered beam considered in this paper is either hardening or softening depending on the ratio $\varepsilon_{1} / \varepsilon_{2}$ [9], which are calculated from values of $\beta_{i}$ given in Eqs. $2-5$. 
In Table 1, some numerical values for the ratio $\left(\varepsilon_{1} / \varepsilon_{2}\right)$ are presented for different values of $C_{r}$ and $C_{t}$ and for the first three modes of vibration.

Table 1. Sample numerical values for the ratio $\left(\varepsilon_{1} / \varepsilon_{2}\right)$ for different values of $C_{r}$ and $C_{t}$ of the first three modes

\begin{tabular}{|c|c|c|c|c|c|c|}
\hline & & & \multicolumn{4}{|c|}{ Rotational Flexibility $C_{r}$} \\
\hline & & & $C_{r}=0$ & $C_{r}=1$ & $C_{r}=5$ & $C_{r}=10$ \\
\hline \multirow{4}{*}{$\begin{array}{l}\text { First } \\
\text { Mode }\end{array}$} & \multirow{4}{*}{$\begin{array}{c}\text { Translational } \\
\text { Rotational } \\
\text { Flexibility } \\
C_{t}\end{array}$} & $C_{t}=0$ & 0.36 & 8 & 56 & 611.1 \\
\hline & & $C_{t}=0.5$ & 2.36 & 8.70 & 33.4 & 299.2 \\
\hline & & $C_{t}=1$ & 2.45 & 11.09 & 30.11 & 176.74 \\
\hline & & $C_{t}=5$ & 2.57 & 30.87 & 46.6 & 93.47 \\
\hline \multirow{4}{*}{$\begin{array}{c}\text { Second } \\
\text { Mode }\end{array}$} & \multirow{4}{*}{$\begin{array}{c}\text { Translational } \\
\text { Rotational } \\
\text { Flexibility } \\
C_{t}\end{array}$} & $C_{t}=0$ & 1.35 & 1.476 & 1 & 2.468 \\
\hline & & $C_{t}=0.5$ & 0.61 & 8.7 & 9.44 & 9.54 \\
\hline & & $C_{t}=1$ & 0.51 & 4.9 & 16.46 & 24.225 \\
\hline & & $C_{t}=5$ & 0.44 & 5.11 & 34.0 & 209.9 \\
\hline \multirow{4}{*}{$\begin{array}{l}\text { Third } \\
\text { Mode }\end{array}$} & \multirow{4}{*}{$\begin{array}{c}\text { Translational } \\
\text { Rotational } \\
\text { Flexibility } \\
C_{t}\end{array}$} & $C_{t}=0$ & 2.95 & 2.48 & 2.4697 & 2.468 \\
\hline & & $C_{t}=0.5$ & 1.63 & 1.80 & 1.40 & 1.28 \\
\hline & & $C_{t}=1$ & 1.63 & 1.78 & 1.35 & 1.22 \\
\hline & & $C_{t}=5$ & 1.63 & 1.76 & 1.32 & 1.19 \\
\hline
\end{tabular}

As an example and to shed more light on the nonlinear dynamics of a tapered beam, results for forced vibration are obtained and presented in Figure 2 for selected values of the physical parameters $C_{r}, C_{t}, \alpha$ and $\delta$. In Figure 2(a), results of the steady state frequency response are obtained for the first mode of vibration for $C_{t}=0, \alpha=0.1, \delta=0.1$ and for the indicated values of $C_{r}$. These results show that the frequency response of the first mode is bent towards the right, i.e. the frequency exhibits a hardening behaviour, when the value of $C_{r}=0$, and the frequency exhibits a softening behaviour " bend to the left" for other values of $C_{r}$. This is due to the fact that when $\varepsilon_{1} / \varepsilon_{2}<1.6$ the behaviour is of hardening type and when $\varepsilon_{1} / \varepsilon_{2}>1.6$ is of a softening type $[16,18]$. For the second mode, the behaviour is of hardening type for all values of $C_{r}$ except the case $C_{r}=10$ (see Figure 2(b)). In Figure 2(c) the behaviour of the third mode is of softening type, for all values of $C_{r}$ and $C_{t}$. Note that in this case the curves for $C_{r}=1, C_{r}=5$ and $C_{r}=10$ have coalesced onto the same curve. 


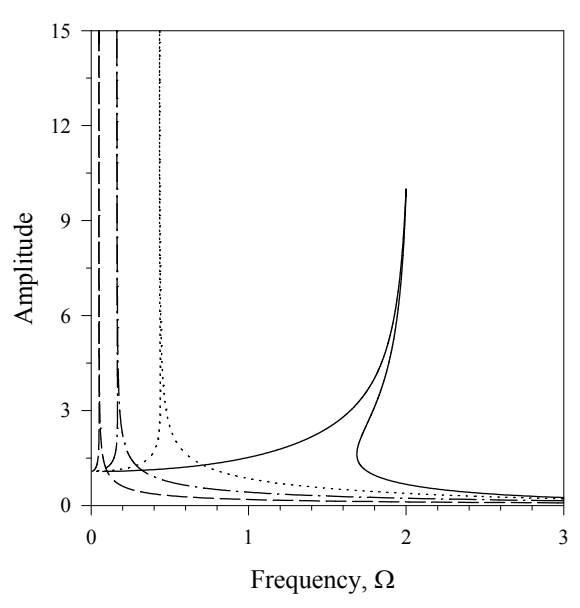

(a) First mode

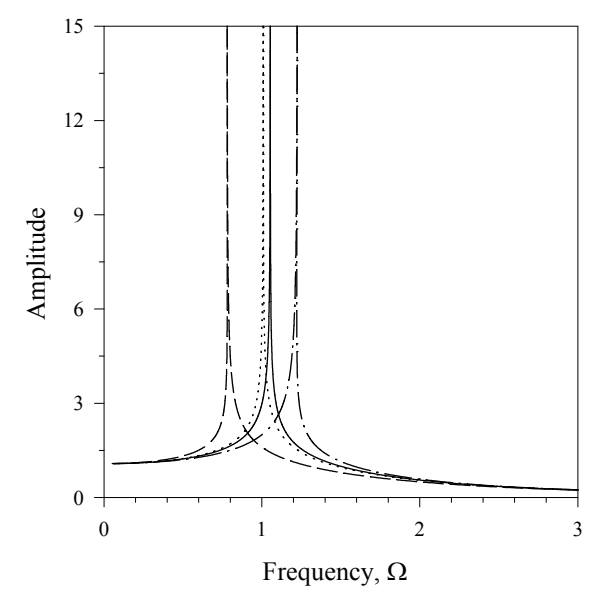

(b) second mode

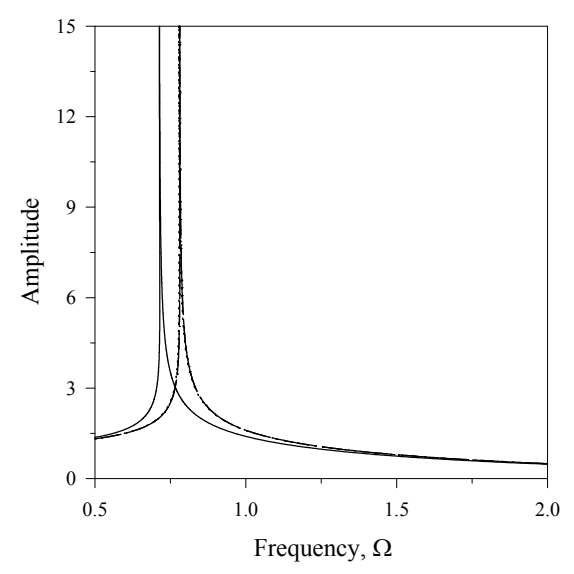

(c) Third mode

Figure 2. Steady State Frequency Responses for $\alpha=0.1, C_{t}=0$ and $C_{r}=0,1,5,10$

$$
\left(C_{r}=0 \longrightarrow, C_{r}=1 \cdots \cdots \cdots \cdot C_{r}=5-\cdots \cdot-\text { and } C_{r}=10----\right)
$$

The stability analysis of the nonlinear oscillator described in Eq. 9 was verified near the principal resonance zone for selected values of system parameters $F_{0}, \varepsilon_{1}, \varepsilon_{1}$ and $\delta$, using computer simulation and with the aid of Time histories, Phase Plane, Poincare Map and Fast Fourier Transforms (FFT).

Results for a softening type oscillators, i.e. $\varepsilon_{1} / \varepsilon_{2}>1.6$, are presented in Figure 3 with the parameters $F_{0}, C_{r}, C_{t}, \alpha$ and $\delta$ chosen to be $5,0.5,10,0.1$ and 0.1 respectively of the second mode of vibration of the elastically restrained tapered beam. 


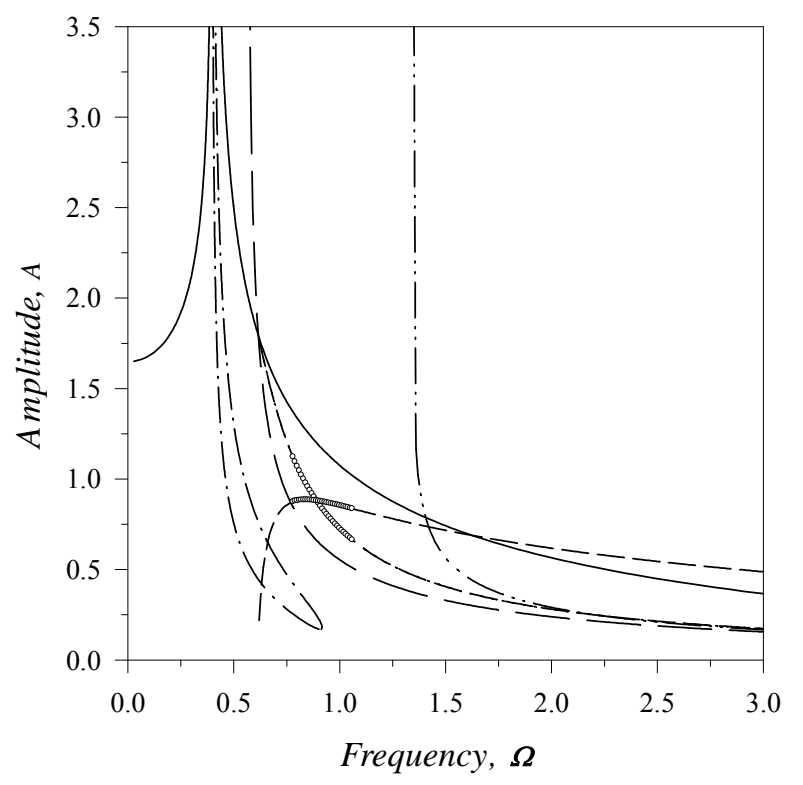

Figure 3. Steady State Frequency Response (SSFR), First Order Stability (1 ${ }^{\text {st }}$ Stab.), Second order Stability ( $2^{\text {nd }}$ Stab.), Biased Solution and Its Stability. $F_{0}=5, C_{t}=0.5, C_{r}=10$ and $\delta=0.1$ for the second mode:

In Figure 3, using the HB method, the steady state response first and second order unstable regions are obtained using a single term only, in addition to the biased solution and its stability given in Eq. 24. It is known that the first order unstable region intersects the steady state response curve at the vertical tangency point. In the same figure the biased solution and its stability are also shown. The dynamics behaviour was verified numerically and steady state numerical solutions were obtained in the resonance area. The steady state response curve enters the second unstable region at $\Omega>1.4$, and the biased solution is unstable at $0.78<\Omega<1.06$, at which there is a possibility of period doubling bifurcation PDB, i.e. the PDB can occur also for $\Omega>1.40$ in addition to range predicted by the stability analysis of the biased solution. Numerical simulations have shown that, by increasing the frequency, the PDB is first observed at $\Omega=0.99$ followed by $3 T$ and $9 T$ attractors at $\Omega=1.04$ and $\Omega=1.055$ respectively, and they develop into chaos at $\Omega=1.11$. The first chaotic zone observed is in the range $1.11<\Omega \leq 1.29$ and then ends by a $3 T$ attractor. The $3 T$ attractor disappears and chaos returns in the range $2.09 \leq \Omega \leq 2.15$, followed by period doubling at $\Omega=2.3$ and higher period doubling, i.e. $4 T$ at $\Omega=2.35$. These period doublings do not develop into chaos. Further investigations showed that a PDB was observed in the range $2.95<\Omega \leq 3.05$ and the periodicity returns to the system for $\Omega>3.1$. In Figures $4-9$ the time histories, phase planes, Poincare maps and FFT are shown for different values of $\Omega$ and for the parameters $\left(F_{\mathrm{o}}=5, C_{t}=0.5, C_{r}=10, \alpha=0.1, \delta=0.1\right)$. 


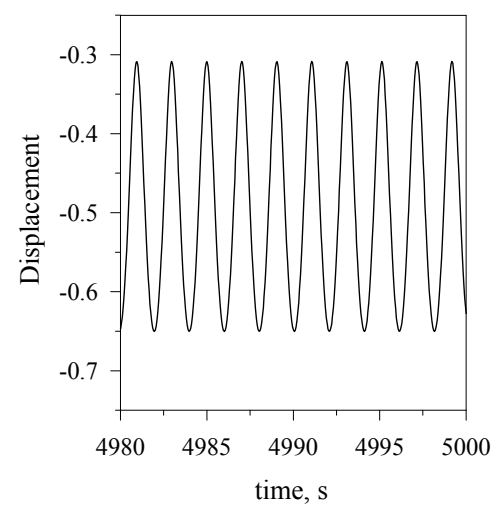

(a) time history

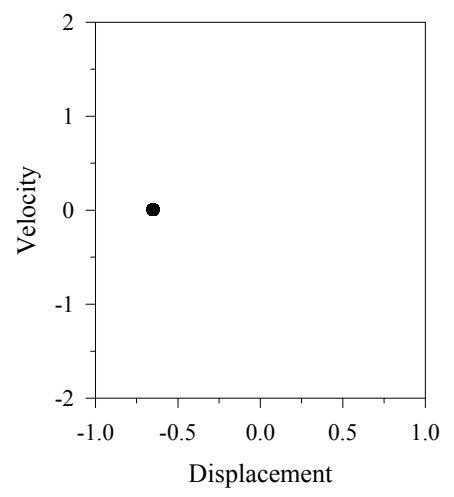

(c) Poincare map

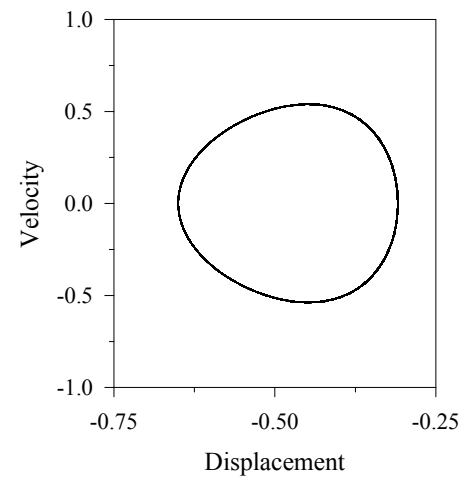

(b) Phase Plane

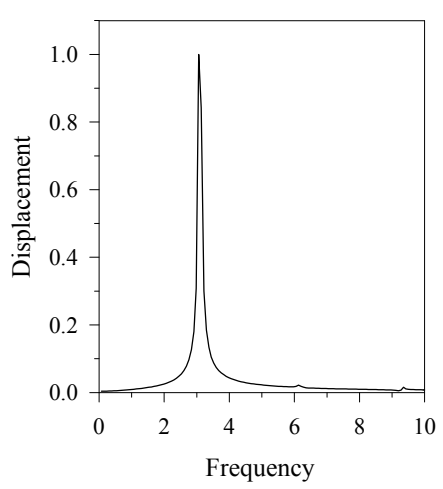

(d) Fast Fourier Transform

Figure 4. Time history, Phase Plane, Poincare map and Fast Fourier Transform (FFT). $F_{0}=5, C_{t}=0.5, C_{r}=10, \delta=0.1$ and $\Omega=3.1$ periodic (1T) for the second mode 


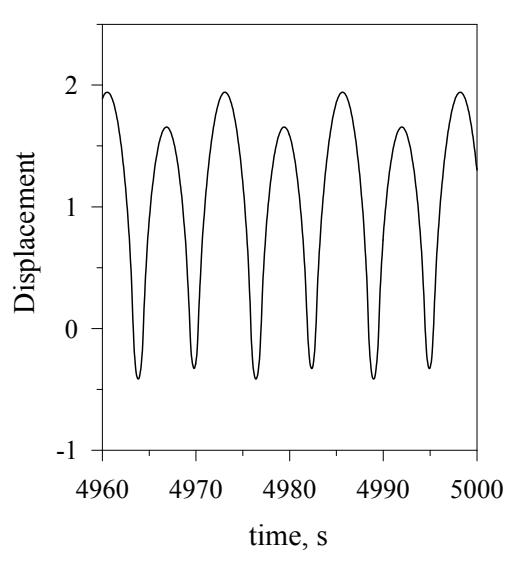

(a) time history

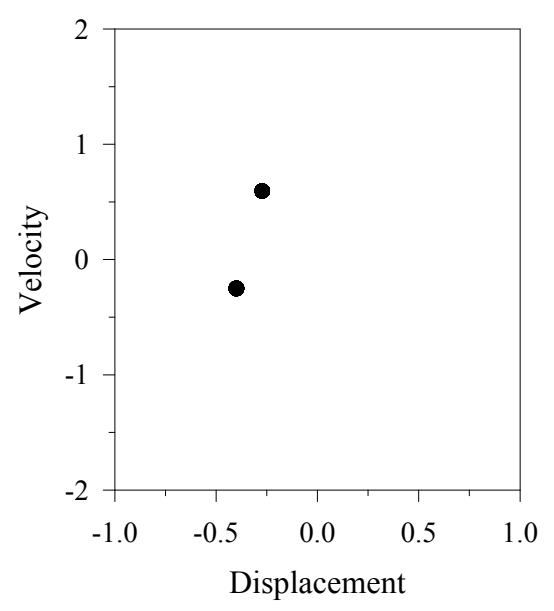

(c) Poincare map

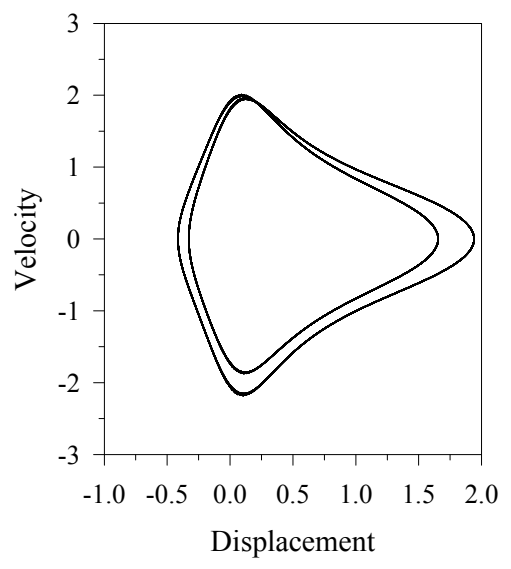

(b) Phase Plane

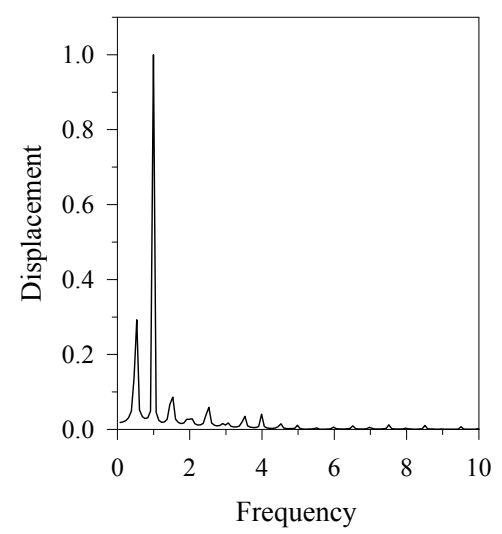

(d) Fast Fourier Transform

Figure 5. Time history, Phase Plane, Poincare map and Fast Fourier Transform (FFT).

$F_{0}=5, C_{t}=0.5, C_{r}=10, \delta=0.1$ and $\Omega=1.0$ periodic doubling (2T) for the second mode 


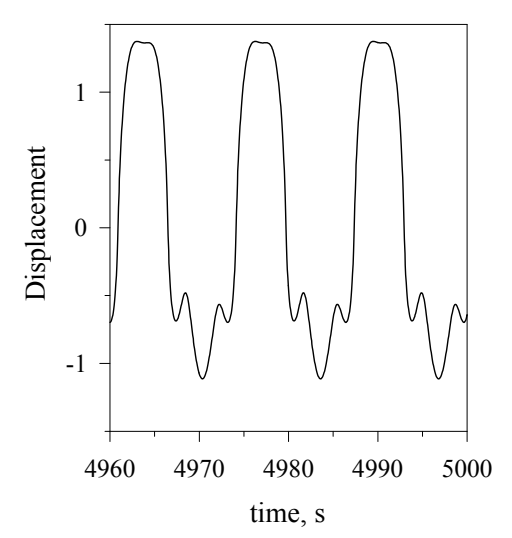

(a) time history

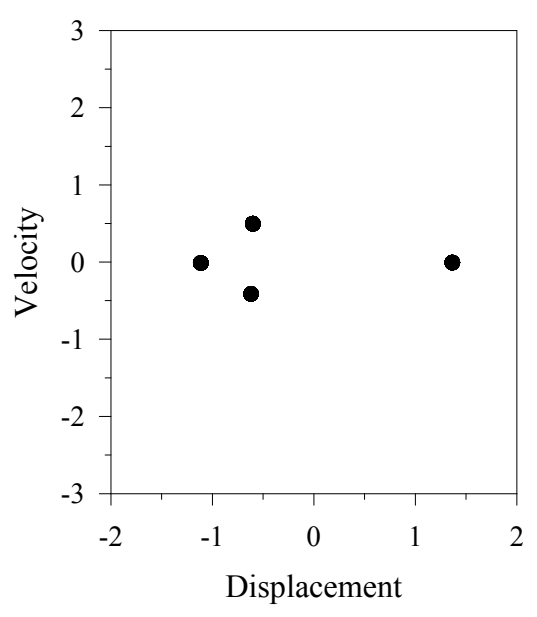

(c) Poincare map

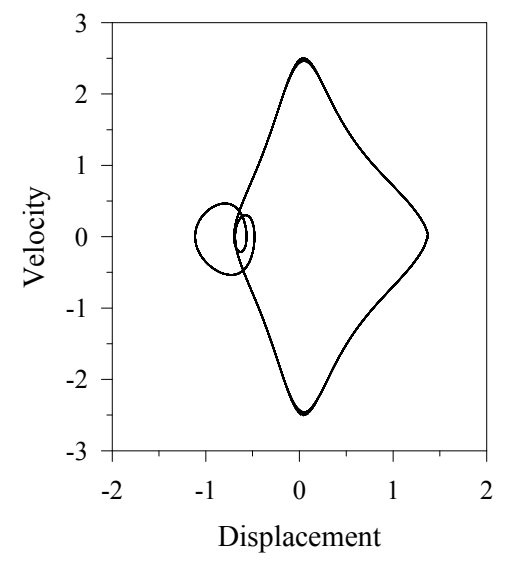

(b) Phase Plane

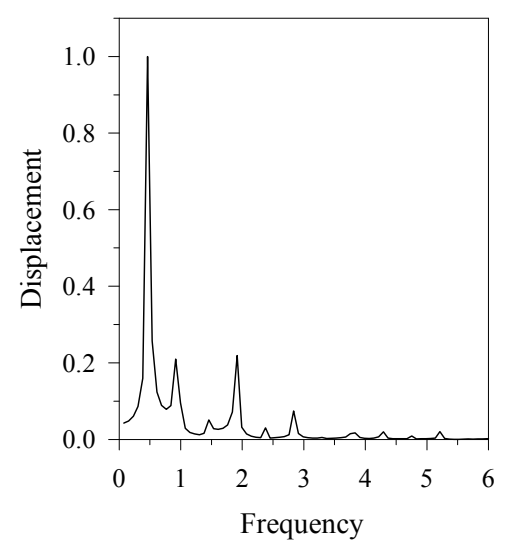

(d) Fast Fourier Transform

Figure 6. Time history, Phase Plane, Poincare map and Fast Fourier Transform (FFT). $F_{0}=5, C_{t}=0.5, C_{r}=10, \delta=0.1$ and $\Omega=1.9$ higher period doubling (4T) for the second mode 


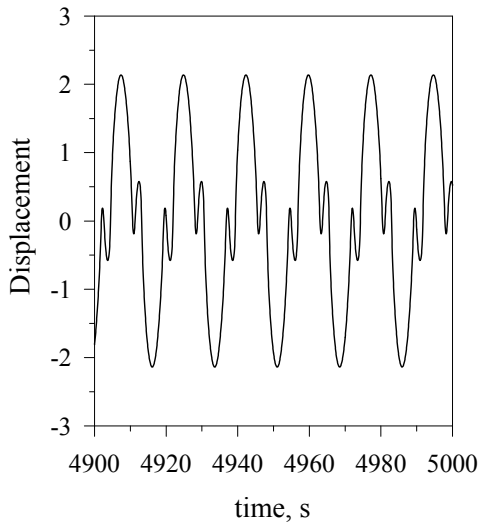

(a) time history

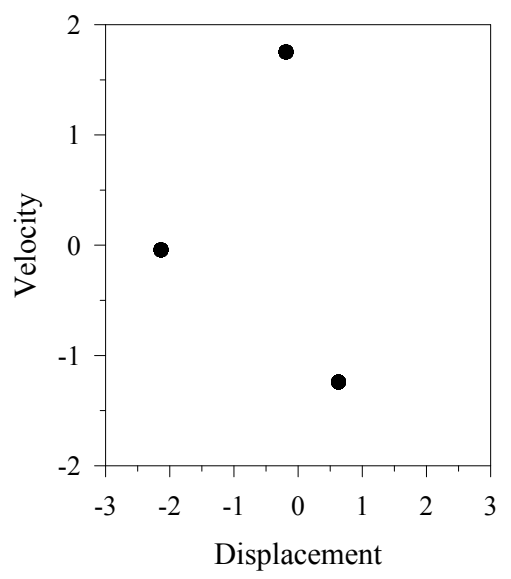

(c) Poincare map

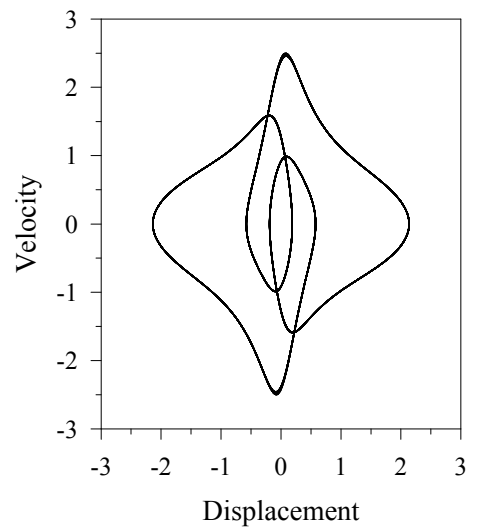

(b) Phase Plane

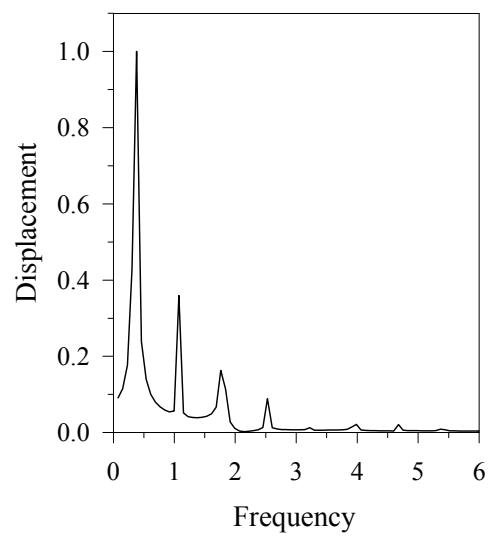

(d) Fast Fourier Transform

Figure 7. Time history, Phase Plane, Poincare map and Fast Fourier Transform (FFT). $F_{0}=5, C_{t}=0.5, C_{r}=10, \delta=0.1$ and $\Omega=1.08$ (3T attractor) for the second mode 


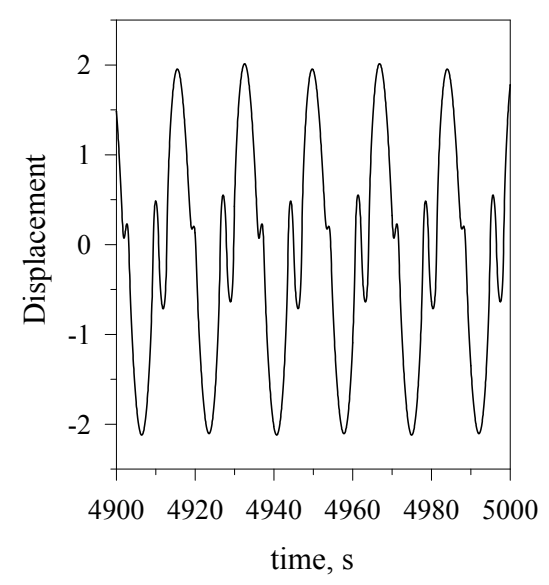

(a) time history

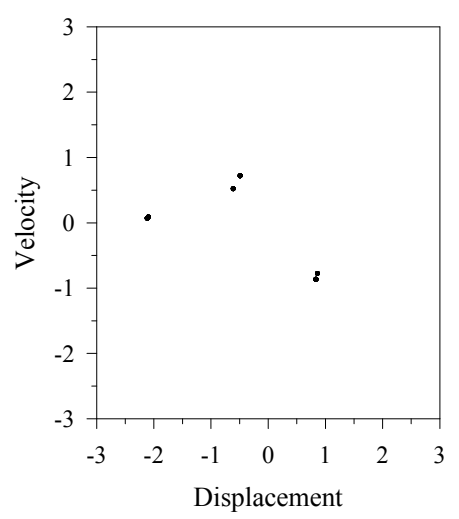

(c) Poincare map

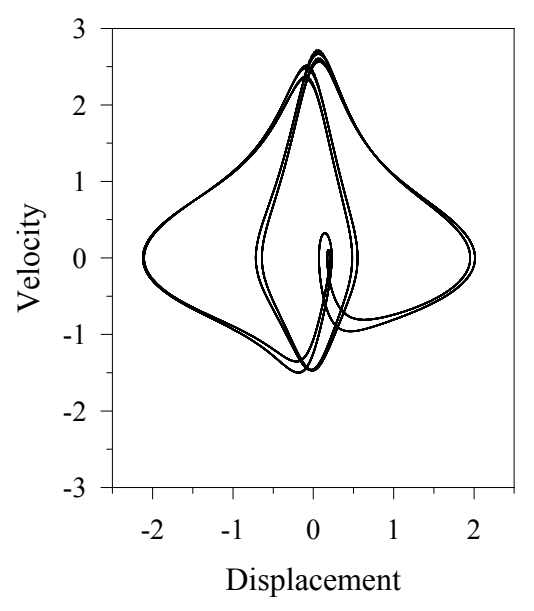

(b) Phase Plane

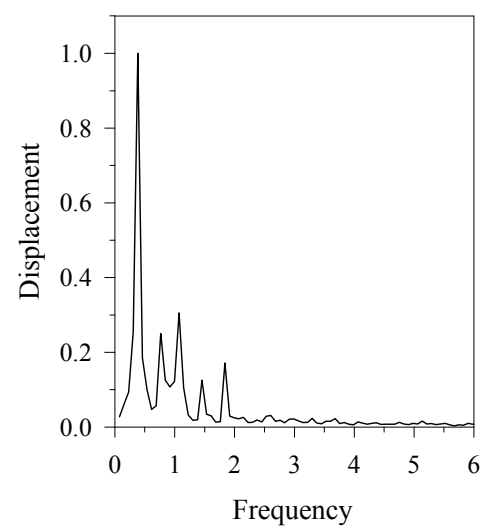

(d) Fast Fourier Transform

Figure 8. Time history, Phase Plane, Poincare map and Fast Fourier Transform (FFT). $F_{0}=5, C_{t}=0.5, C_{r}=10, \delta=0.1$ and $\Omega=1.1$ (6T attractor) for the second mode 


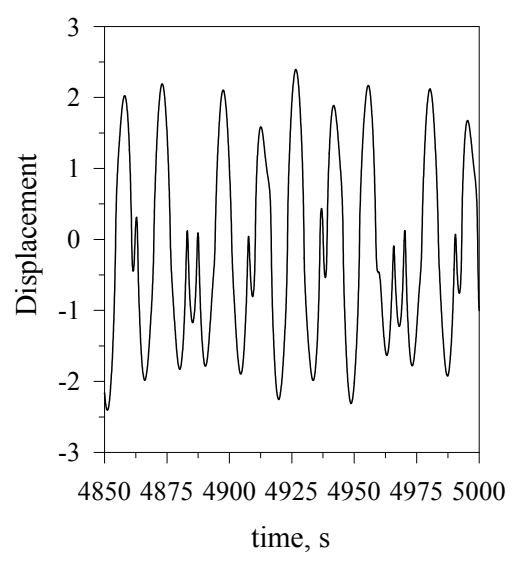

(a) time history

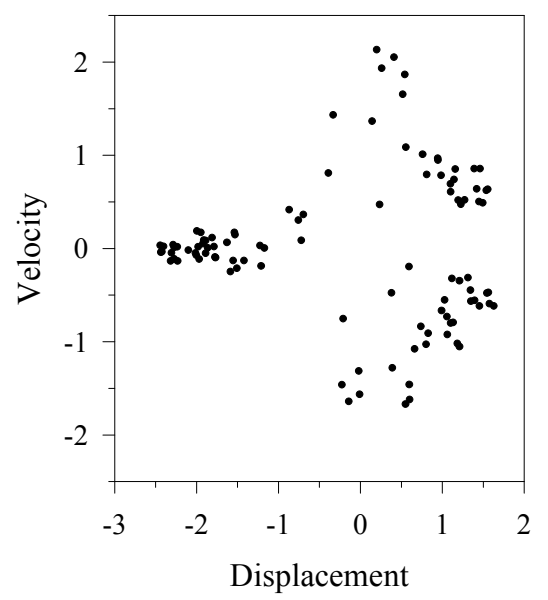

(c) Poincare map

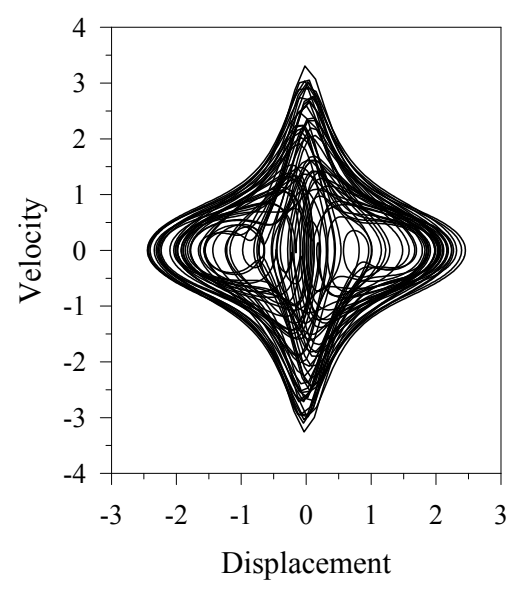

(b) Phase Plane

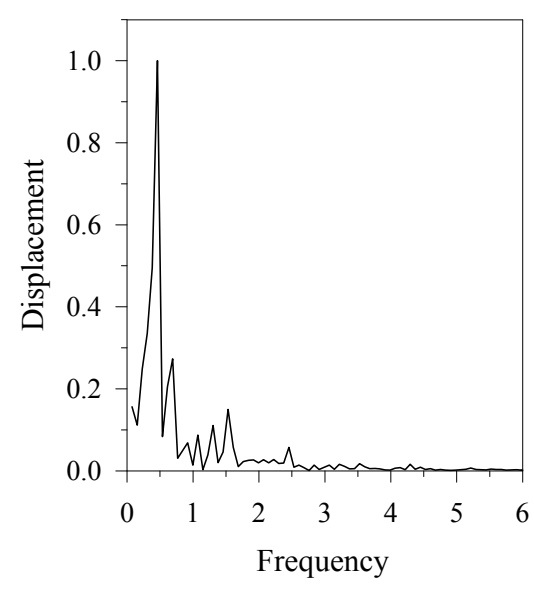

(d) Fast Fourier Transform

Figure 9. Time history, Phase Plane, Poincare map and Fast Fourier Transform (FFT). $F_{0}=5, C_{t}=0.5, C_{r}=10, \delta=0.1$ and $\Omega=1.29$ (Chaotic behaviour) for the second mode

Results presented in Figure 3 and with the aid of computer simulations (Figures 4-9) show that, as one may expect, the resonance curves of the asymmetric solution intersect those of the symmetric solution near the region of chaotic motion, which lies in the zone where the principal resonance curves of the symmetric solution may enter the second unstable region. Results for a hardening type oscillator, i.e. $\varepsilon_{1} / \varepsilon_{2}<1.6$, are presented in Figure 10 with the parameters $F_{0}=35, C_{r}=5$, $C_{t}=1, \alpha=0.1$ and $\delta=0.1$, for the third mode of vibration of the tapered beam under consideration. In this figure, the steady state response, first order and second unstable boundaries, biased solution and stability of the biased solution "Asymmetric" are also presented for the nonlinear oscillator with hardening characteristics. The first order unstable region intersects the response curve at the point of vertical tangency and the second unstable region has two boundaries; the first one is located at the super-harmonic resonance zone at $0.6<\Omega<0.635$ and the second intersects with the response curves at $\Omega=2.42$. From the figure, the asymmetric solution intersects with symmetric one at $\Omega \cong 1.60$, i.e. before the steady state response enters the second 
boundary of the second unstable region. In addition the biased solution is unstable at $1.91<\Omega<2.19$, at which there is a possibility of period doubling bifurcation (PDB). Results obtained, but not shown for the sake of brevity, indicated that the PDB and chaos may occur inside the regions $0.6<\Omega<0.635, \Omega>2.42$ and $1.91<\Omega<2.19$, at which the PDB may develop into higher period doublings and then culminates into chaos.

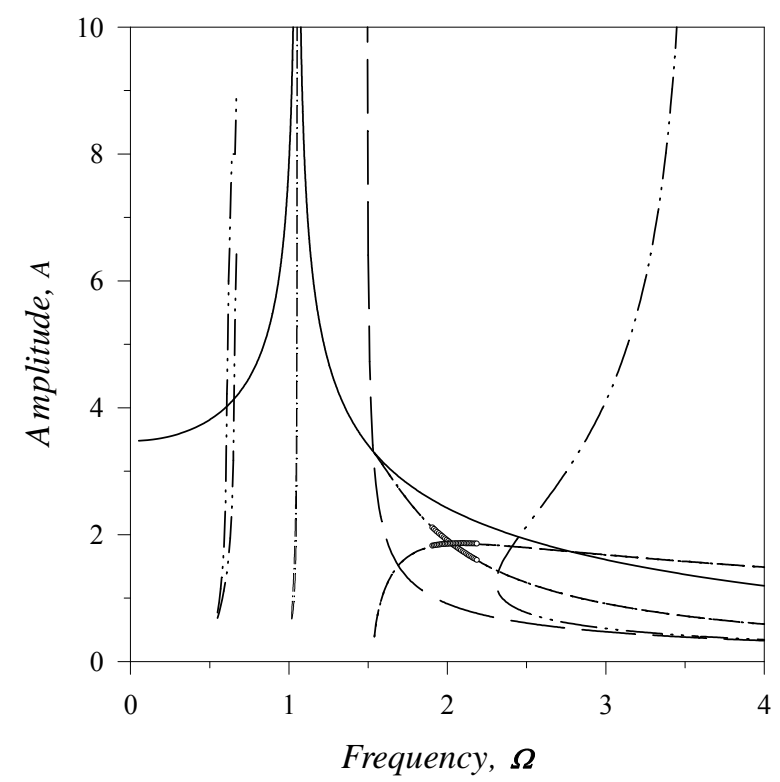

Figure 10. Steady State Frequency Response (SSFR), First Order Stability ( $1^{\text {st }}$ Stab.), Second order Stability ( $2^{\text {nd }}$ Stab.), Biased Solution and Its Stability. $F_{0}=35, C_{t}=1, C_{r}=5$ and $\delta=0.02$ for the third mode: Steady State Frequency Response (SSFR), First Order Stability ( $1^{\text {st }}$ Stab.), Second order Stability ( $2^{\text {nd }}$ Stab.),

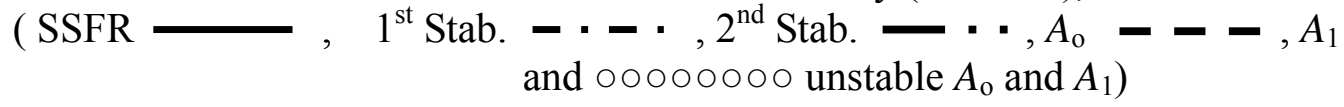

\section{CONCLUSIONS}

The present work has studied the steady state frequency response of an elastically restrained cantilever tapered beam. The nonlinear uni-modal equation of motion was taken from [14]. The steady state responses under a sinusoidal excitation were obtained for different values of physical parameter; rotational flexibility $C_{r}$, translational flexibility $C_{t}$, taper ratio $\alpha$, damping $\delta$ and excitation level $F_{0}$. The steady state frequency response curves are presented for the first three modes of vibration using the method of harmonic balance.

It was shown that the variation of $C_{r}$ and $C_{t}$ is associated with a qualitative change in the dynamics behaviour of the steady state response.

It has been shown that the frequency response curves of the asymmetric solution intersect those of the symmetric solution before the symmetric solutions penetrate into the second unstable region, regardless the characteristic type of the nonlinear equation of motion, i.e. softening or hardening. In addition, it has been shown that at the point of intersection between asymmetric and symmetric 
solutions, i.e. when $A_{0}$ in the biased solution has non-zero real value, the symmetric solution bifurcates into an asymmetric one and the PDB appears in this area and in some cases the PDB developed into chaos.

\section{ACKNOWLEDGMENT}

A.A. Al-Qaisia and M. S. Abdel-Jaber acknowledge the support of the Deanship of Scientific Research, The University of Jordan, Amman-Jordan.

\section{REFERENCES}

[1] Kanga, J. H. and Leissa, A. W. "Three-dimensional Vibration Analysis of Thick, Tapered Rods and Beams with Circular Cross-section”, International Journal of Mechanical Sciences, 2004, Vol. 46, pp. 929-944.

[2] Andrade, A., Providência, P. and Camotim, D. "Elastic Lateral-torsional Buckling of Restrained Web-tapered I-beams", Computers and Structures, 2010, Vol. 88, pp. 1179-1196.

[3] Das, D., Sahoo, P. and Saha, K. "Out-of-plane Free Vibration Analysis of Rotating Tapered Beams in Post-elastic Regime", Materials and Design, 2009, Vol. 30, pp. 2875-2894.

[4] Hoseini, S. H., Pirbodaghi, T., Ahmadian, M. T., and Farrahi, G. H. "On the Large Amplitude Free Vibrations of Tapered Beams: an Analytical Approach", Mechanics Research Communications, 2009, Vol. 36, pp. 892-897.

[5] De Rosa, M.A., Lippiello, M., Maurizi, M. J. and Martin, H. D., "Free Vibration of Elastically Restrained Cantilever Tapered Beams with Concentrated Viscous Damping and Mass", Mechanics Research Communications, 2010, Vol. 37, pp. 261-264.

[6] Cheng, Y. Yu, Z., Wub, X. and Yuan Y., "Vibration Analysis of a Cracked Rotating Tapered Beam using the p-version Finite Element Method", Finite Elements in Analysis and Design, 2011, Vol. 47, pp.825-834.

[7] Ozgumus, O.O. and Kaya, M.O., "Energy Expressions and Free Vibration Analysis of a Rotating Double Tapered Timoshenko Beam Featuring Bending-torsion Coupling", International Journal of Engineering Science, 2007, Vol. 45, pp. 562-586.

[8] Auciello, N.M. and Nole, G. "Vibrations of a Cantilever Tapered Beam with varying section properties and Carrying a Mass at the Free End," Journal of Sound and Vibration, 1998, Vol. 214, pp. 105-119.

[9] Nagaya, K. and Hai, Y. "Seismic Response of Underwater members of Variable Cross Section", Journal of Sound and Vibration, 1985, Vol. 119, pp. 119-138.

[10] Laura, P.A.A. and Gutierrez, R.H. "Vibrations of an Elastically restrained Cantilever Beam of Varying Cross Sections with Tip Mass of Finite Length", Journal of Sound and Vibration, 1986, Vol. 108, pp.123-131.

[11] Shong, J.W. and Chen, C.T. "An Exact Solution for the Natural Frequency and Modes Shapes of an Immersed Elastically Wedge Beam Carrying an Eccentric Tip Mass with Mass Moment of Inertia", Journal of Sound and Vibration, 2005, Vol. 286, pp. 549-568.

[12] Chen, D.W. and Wu, J.S. "The Exact Solutions for the Natural Frequency and Modes Shapes of Non-Uniform Beams with Multiple Spring-Mass Systems", Journal of Sound and Vibration, 2008, Vol. 313, pp. 772-783.

[13] Abdel-Jaber, M.S., Al-Qaisia, A.A., Abdel-Jaber, M. and Beale R.G., Nonlinear Natural Frequencies of an elastically restrained tapered beam, Journal of Sound and Vibration, 2008, Vol. 255, pp. 299-322.

[14] Abdel-Jaber, M., Al-Qaisia, A.A. and Abdel-Jaber, M.S."Non-linear natural frequencies of a tapered cantilever beam”, Advanced Steel Construction, 2009, 5, pp259-272. 
[15] Al-Qaisia, A.A. and Hamdan, M.N. "Bifurcation and Chaos of an Immersed Cantilever Beam in a Fluid and Carrying an Intermediate Mass", Journal of Sound and Vibration, 2002, Vol. 253, pp. 859-888.

[16] Al-Qaisia, A.A. and Hamdan, M.N. "On the Steady State Response of Oscillators with Static and Inertia Non-Linearities", Journal of Sound and Vibration, 1999, Vol. 223, pp. 49-71.

[17] Al-Qaisia, A.A. and Hamdan, M.N., "Bifurcations of Approximate Harmonic Balance Solutions and Transition to Chaos in an oscillator with Inertial and Elastic Symmetric Nonlinearities", Journal of Sound and Vibration, 2001, Vol. 244, pp. 453-479.

[18] Al-Qaisia, A.A. and Hamdan, M.N. and Al-Bedoor, B., "On the Steady State Response of a Cantilever Beam Partially Immersed and Carrying an Intermediate Mass", Shock and Vibration, 2000, Vol. 7, pp. 179-194. 